\title{
Electron microscopic findings of parallel tubular granules in a case of chronic neutrophilic leukaemia
}

\author{
N Takemori, K Hirai, R Onodera, N Saito, M Namiki
}

\begin{abstract}
A case of chronic neutrophilic leukaemia (CNL) is reported. The diagnosis was based on leucocytosis with mature neutrophils, a raised leucocyte alkaline phosphatase score, negative Philadelphia chromosome, and extensive infiltration of neutrophils in various differentiation stages into the bone marrow. When viewed by light microscopy, these neutrophils were almost normal in appearance, except for the presence of ring shaped nuclei and cytoplasmic vacuoles. Electron microscopy showed that neutrophilic promyelocytes and early myelocytes in the bone marrow frequently possessed unique rounded granules consisting of clustered parallel tubules, of 29-31 nm in diameter, and occasional amorphous electron dense material. These parallel tubules showed a hexagonal array; the granules were termed parallel tubular granules (PTGs). PTGs were positive for electron microscopic myeloperoxidase, and were seen exclusively in neutrophilic promyelocytes and early myelocytes.
\end{abstract}

These findings suggest that PTGs represent certain primary granules peculiar to immature neutrophils. Their presence might also be helpful in diagnosing CNL.

(F Clin Pathol 1994;47:367-369)

Chronic neutrophilic leukaemia (CNL) is a rare disease in which mature neutrophils increase substantially in the peripheral blood. Until the discovery of the Philadelphia chromosome in 1960 , this disease was regarded as a subtype of chronic myeloid leukaemia (CML). Subsequent chromosomal studies, however, have disclosed that most of the CML cases were positive for the Philadelphia chromosome. Thus the disease state showing persistent leucocytosis with predominant mature neutrophils, an increased leucocyte alkaline phosphatase score, and no Philadelphia chromosome has been regarded as a disease distinct from CML. After the discovery of the Philadelphia chromosome, 48 cases of CNL had been documented by $1987 .{ }^{1}$ In a recent opportunity to treat a patient with CNL, it was found that immature myeloid cells in the bone marrow possessed unique rounded granules consisting of clustered parallel tubules with occasional amorphous electron dense material. These granules were named parallel tubular granules (PTGs). To the authors' knowledge, this is the first report of CNL with PTGs.

\section{Case report}

A 70 year old man who had persistent thrombocytopenia of less than $60 \times 10^{6} / 1$ for nine months was admitted to the Asahikawa Medical College Hospital, Japan, in May 1992 for extensive leucocytosis. The results of peripheral blood tests on admission were: white cell count $41 \times 10^{9} / 1$ (neutrophil count $34 \times 10^{9} / 1$ ), red blood cell count $4.9 \times 10^{12} / 1$, and platelet count $21 \times 10^{9} / 1$. A bone marrow aspirate on admission showed extensive infiltration of neutrophilic myeloid cells in various differentiation stages. Raised concentrations of serum lysozyme and vitamin $\mathbf{B}_{12}$ were present. The patient had neither splenomegaly nor generalised lymph node swelling. Neither inflammatory lesions nor tumors which cause leucocytosis were present. Chromosomal analyses performed on several occasions consistently showed a normal karyotype. No rearrangement of the breakpoint cluster region (bcr) gene was detected by Southern blot analysis using bone marrow cell DNA. The leucocyte alkaline phosphatase score was increased (patient 321; control 281). Mature neutrophilic leucocytosis persisted until the initiation of chemotherapy. Thus the patient was diagnosed as having CNL. In this case a temporary reduction of leucocyte count was achieved by administering carboquone combined with hydroxyurea; but the treatment was stopped because of severe thrombocytopenia. For the treatment of this, $60 \mathrm{mg}$ Cepharanthin (a complex of biscoclaurine alkaloids including cepharanthine and its analogues, which were extracted from Stephania cepharantha Hayata, Kaken Shoyaku Co. Ltd, Tokyo, Japan) was given daily, starting in August 1992. This treatment was fairly effective, resulting in an increase in platelet count. However, leucocytosis continued to advance gradually, reaching over $200 \times 10^{9} / 1$ in late December 1992. Then, the patient was given a single injection of ranimustine combined with the daily Cepharanthin treatment. This treatment was effective, and peripheral leucocyte counts decreased substantially without additional chemotherapy. At the time of writing, the patient remains in a fairly satisfactory condition with a white cell count of less than $5 \times 10^{9} / 1$, platelet count of more than $60 \times$ $10^{9} / 1$, and haemoglobin of more than $130 \mathrm{~g} / 1$. 


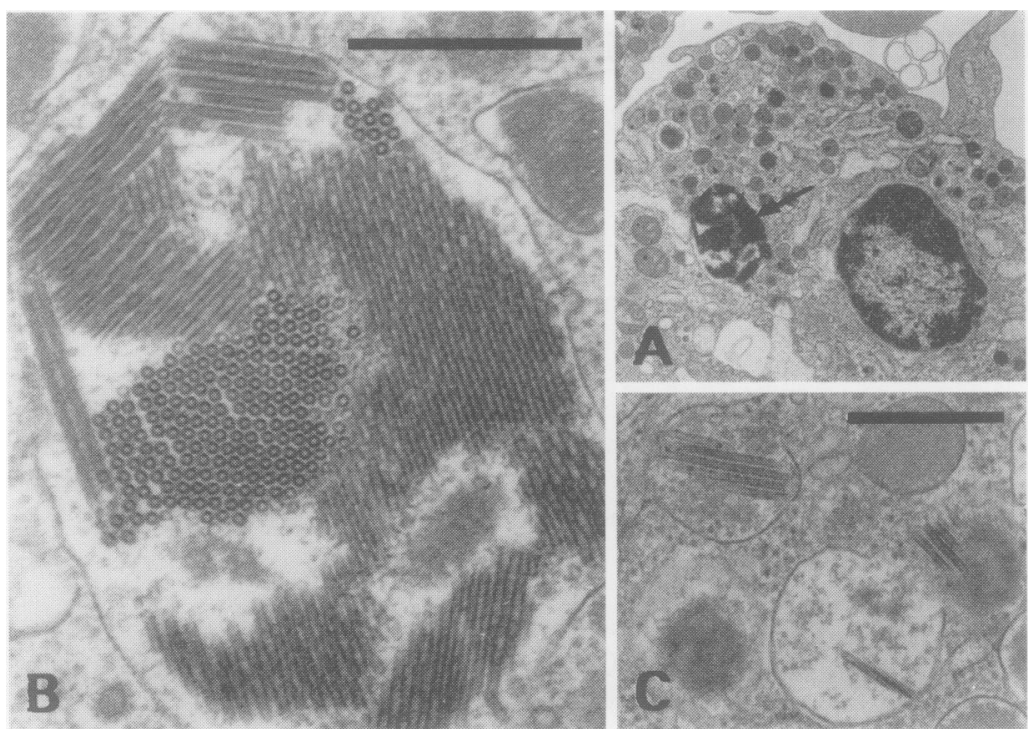

Figure 1 Electron micrograph showing PTGs. (A) Neutrophilic promyelocyte containing a well developed PTG (arrow); (B) higher power magnification of the PTG. The PTG comprises clustered parallel tubules showing a hexagonal array. Bar indicates $500 \mathrm{~nm}$. (C) Early PTGs seen in the cytoplasm of a neutrophilic promyelocyte; they contain only a few tubules. Bar indicates $500 \mathrm{~nm}$.

Bone marrow aspirations were carried out on several occasions during his inpatient stay. For light microscopy, May-Grünwald Giemsa (MGG) staining and various conventional cytochemical stainings were carried out using bone marrow smears. For electron microscopy, aspirated bone marrow particles were routinely processed according to the method previously reported. ${ }^{2}$ Ultrathin sections (20-30 nm thick) were stained with uranyl acetate and lead citrate, and observed with a Hitachi H-800 electron microscope at an accelerating voltage of $75 \mathrm{kV}$. Electron microscopic myeloperoxidase (MPO) staining was also carried out at the same time. To demonstrate clearly reaction products, ultrathin sections were observed without staining. As a control, hydrogen peroxide was omitted from the incubation medium.
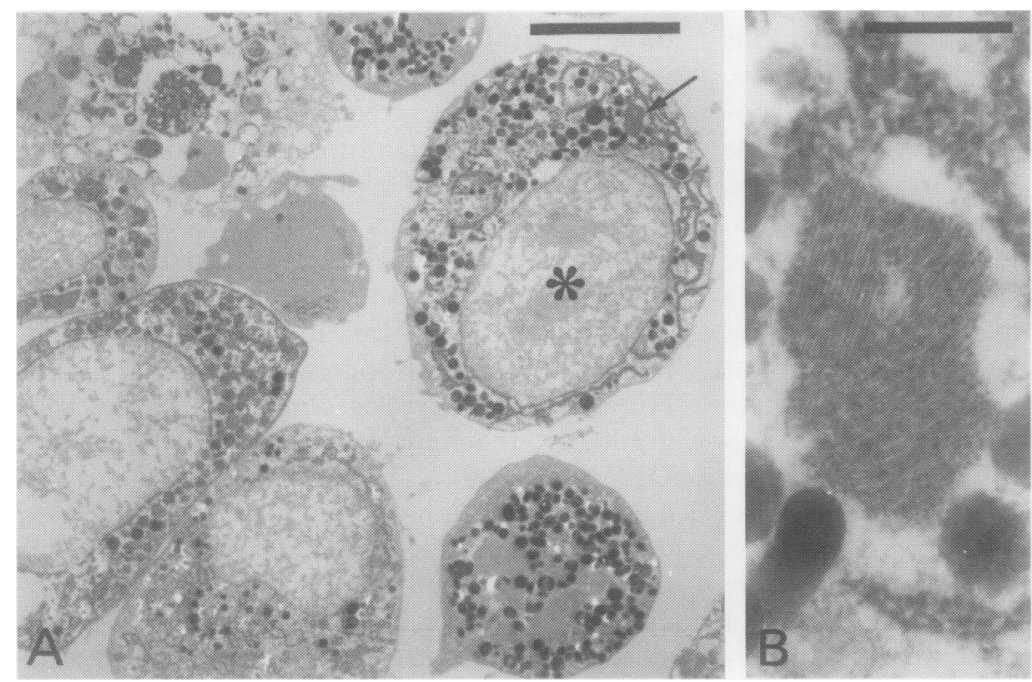

Figure 2 Electron micrograph of bone marrow showing myeloperoxidase activity. (A) Extensive myeloperoxidase activity is seen in neutrophils. One neutrophilic promyelocyte $(\star)$ has a well developed PTG (arrow) in the cytoplasm. The bar indicates $5 \mu \mathrm{m}$. (B) Higher magnification of the PTG indicated by the arrow. The PTG is positive for myeloperoxidase. The bar indicates $500 \mathrm{~nm}$.

\section{Results}

Peripheral blood smear preparations during leucocytosis showed increased mature neutrophils, accounting for more than $80 \%$ of peripheral leucocytes. Bone marrow smear preparations consistently showed substantial infiltration of neutrophilic myeloid cells in various differentiation stages. Some neutrophils had occasional ring shaped nuclei and cytoplasmic vacuoles. However, most of the neutrophils were almost normal in appearance. Immature neutrophils, including promyelocytes and early myelocytes, had occasional coarse azurophilic granules. No Auer bodies were seen in leukaemic cells in the bone marrow or peripheral blood. Electron microscopy showed that neutrophilic promyelocytes and early myelocytes in the bone marrow frequently possessed unique rounded or oval granules, of $1-3 \mu \mathrm{m}$ in diameter, consisting of clustered parallel tubules with occasional amorphous electron dense material (PTGs). Transverse sections of PTGs showed that the tubules, of 29$31 \mathrm{~nm}$ in diameter, were arranged in a hexagonal pattern. Each tubule had a lumen of about $10-13 \mathrm{~nm}$ in diameter and a wall of about $8-9 \mathrm{~nm}$ in thickness. The distance from centre to centre of two neighbouring tubules was about $34-36 \mathrm{~nm}$. PTGs in various developmental stages were also frequently present in these cells. Early PTGs looked like small rounded granules $(0.4-0.6 \mu \mathrm{m}$ in diameter) with a few tubules (fig 1). Intermediate granules between mature and early PTGs were also present. Neutrophilic metamyelocytes, band neutrophils, and segmented neutrophils contained no PTGs. No PTGs were present in the bone marrow cells, with the exception of neutrophils. Electron microscopic MPO staining showed that these granules were positive for MPO (fig 2). No positive reaction products were seen in the control. In this case PTGs were seen exclusively in the bone marrow obtained during the initiation of Cepharanthin treatment.

\section{Discussion}

With respect to the diagnosis of CNL, the following criteria are necessary: ${ }^{1}$ (1) severe sustained mature neutrophilic leucocytosis $>20 \times 10^{9} / 1$; (2) the absence of Philadelphia chromosome; (3) the absence of other chronic myeloproliferative disorders (polycythaemia vera and essential thrombocythaemia); (4) a high leucocyte alkaline phosphatase score; (5) the absence of a reactive leukemoid state.

In this case, all the criteria described above were satisfied. Furthermore, no rearrangement of the $b c r$ gene was detected. Neutrophils with ring shaped nuclei are known to represent one of the features characteristic of CNL. ${ }^{3}$ In this case these neutrophils persisted in the peripheral blood as well as in the bone marrow.

The most striking finding in this case was the presence of PTGs in neutrophilic promyelocytes and early myelocytes. As far as 
the authors are aware, there has been no previous description of such structures in human neutrophilic myeloid cells. The fact that PTGs were positive for MPO at the electron microscopic level seems to indicate that they represent primary granules. In this case PTGs in various developmental stages were exclusively seen in neutrophilic promyelocytes and early myelocytes. The presence of PTGs in these cells combined with their MPO positivity means that PTGs probably correspond to azurophilic granules.

Auer bodies are seen in leukaemic cells of various types of acute myeloid leukaemia. In particular, they are seen in acute promyelocytic leukaemia (APL) (FAB-M3), consisting of parallel tubules showing a hexagonal array. ${ }^{4}$ PTGs somewhat resemble Auer bodies seen in APL because both structures comprise parallel tubules arranged in a hexagonal pattern. However, the diameter of individual tubules in PTGs ranged from 29 to $31 \mathrm{~nm}$, whereas the diameter of tubules in Auer bodies reported by Breton-Gorius and Houssay ${ }^{4}$ was about $16.5 \mathrm{~nm}$. There is a considerable difference in the diameter of tubules between PTGs and Auer bodies. To confirm this difference, the authors recently examined Auer bodies in leukaemic cells obtained from two patients with APL by electron microscopy. As demonstrated by Breton-Gorius and Houssay, ${ }^{4}$ tubules in Auer bodies were half the diameter of those in PTGs. In the present case no Auer bodies were seen on bone marrow or peripheral blood smears by light microscopy, although PTGs were frequently seen in promyelocytes and early myelocytes by electron microscopy. These findings seem to indicate that PTGs are different in nature from Auer bodies. Dixon et $a l^{6}$ reported a case of undifferentiated APL, in which only a limited number of promyelocytes showed Auer bodies. They analysed azurophilic granules in leukaemic promyelocytes by electron microscopy, applying high angle specimen tilting. They found that azurophilic granules included Auer body precursors and intermediate forms. But the Auer body precursors demonstrated by Dixon et $a l^{6}$ consisted mainly of membranous lamellae. Thus the possibility that PTGs represent Auer body precursors also seems unlikely.

Cepharanthin is known to be effective for increasing platelet counts, particularly in patients with idiopathic thrombocytopenic purpura (ITP). ${ }^{7}$ In this case PTGs were seen exclusively in the bone marrow specimens obtained during Cepharanthin treatment. Thus we can not neglect the possibility that Cepharanthin was related to the formation of PTGs. To confirm such a possibility, we investigated the bone marrow of several patients with ITP, similarly treated with Cepharanthin, by electron microscopy. However, we found that no PTGs were present in myeloid cells of the bone marrow in these patients. PTGs were not related to the ranismustine treatment because they were seen in the bone marrow before this was started.

In conclusion, PTGs were frequently seen in immature neutrophils in a case of CNL by electron microscopy. They seem to represent certain primary granules because they were positive for MPO. As extensive electron microscopic studies have not been performed thus far in cases of CNL, the investigation of other CNL cases is required to determine whether PTGs could be a marker for CNL.

We thank D Hamilton for helpful comments and $\mathrm{K}$ Miyakawa and $M$ Yamamoto for their technical assistance.

1 Miura A, Takahashi T, Nishinari T. Chronic neutrophilic leukemia. Rinsho Ketsueki (Fpn f Clin Hematol) 1987;28: 505-12.

2 Takemori $\mathrm{N}$, Hirai $\mathrm{K}$, Onodera $\mathrm{R}$, et al. Disseminated intravascular coagulation in a patient with acute myeloid leukemia. Ultrastructural evidence of hypercoagulation in bone marrow. Am $\mathcal{f}$ Clin Pathol 1993;99:695-701.

3 Kanoh T, Saigo K, Yamagishi M. Neutrophils with ringshaped nuclei in chronic neutrophilic leukemia. $A m \mathcal{F}$ Clin Pathol 1986;86:748-51.

4 Breton-Gorius J, Houssay D. Auer bodies in acute promyelocytic leukemia. Demonstration of their fine structure and peroxidase localization. Lab Invest 1973;28 $135-41$.

5 Tulliez M, Breton-Gorius J. Three types of Auer bodies in acute leukemia. Visualization of their protein by negative contrast after peroxidase cytochemistry. Lab Invest tive contrast after

6 Dixon BR, Mukherjee TM, Ho JQK. The ultrastructural identification of Auer body precursors in a case of acute promyelocytic leukemia using high-angle specimen tilt. promyelocytic leukemia using high-

7 Kobayashi M, Katayama T, Ochiai S, et al. High-dose cepharanthin therapy for idiopathic thrombocytopenic purpura. Rinsho Ketsueki (Fpn $\mathcal{F}$ Clin Hematol) 1992 33:405-7. 